\title{
Frontières
}

\section{Oui, je suis là}

\section{Diane Laflamme}

Volume 17, numéro 1, automne 2004

\section{Au péril de l'accompagnement}

URI : https://id.erudit.org/iderudit/1073598ar

DOI : https://doi.org/10.7202/1073598ar

Aller au sommaire du numéro

\section{Éditeur(s)}

Université du Québec à Montréal

ISSN

1180-3479 (imprimé)

1916-0976 (numérique)

Découvrir la revue

Citer ce document

Laflamme, D. (2004). Oui, je suis là. Frontières, 17(1), 3-7.

https://doi.org/10.7202/1073598ar

Ce document est protégé par la loi sur le droit d'auteur. L'utilisation des services d'Érudit (y compris la reproduction) est assujettie à sa politique d'utilisation que vous pouvez consulter en ligne.

https://apropos.erudit.org/fr/usagers/politique-dutilisation/
Cet article est diffusé et préservé par Érudit.

Érudit est un consortium interuniversitaire sans but lucratif composé de l’Université de Montréal, l'Université Laval et l'Université du Québec à Montréal. Il a pour mission la promotion et la valorisation de la recherche. https://www.erudit.org/fr/ 
Diane Laflamme, Ph.D., rédactrice en chef.

C'est avec une grande sérénité que j'avais choisi, depuis quelque temps déjà, les quatre mots qui servent de titre à cet éditorial sur les périls de l'accompagnement. Cette sérénité s'est effritée à mesure que je regardais les images que nous a confiées Stephan Hachemi, le fils de la photo-journaliste Zahra Kazémi, assassinée à Téhéran en 2003.

Il y a toujours eu sur la planète des gens mieux outillés que d'autres pour apporter la mort à leurs semblables. Quand ils laissent voir leur visage et leur arsenal, comment ne pas reculer en disant: «Non, j'ai trop peur pour rester là!» En publiant dans ce numéro les photos de Zahra Kazémi, Frontières espère accompagner dans leur deuil la famille et les amis de cette femme qui n'a pas survécu aux périls qu'elle a affrontés pour accompagner le cheminement de ses compatriotes emprisonnés. Ses photos disent: «Oui, je suis là » dans des lieux où notre regard a souvent peur de s'aventurer, malgré la protection dont nous jouissons confortablement installés devant nos téléviseurs. Ces photos montrant le visage et les réalisations de ceux qui sont eux-mêmes des dangers de mort pour autrui vous accompagneront à mesure que vous parcourrez nos pages: à vous la position inconfortable de l'accompagné-e. En fin de parcours, à la rubrique Regard, vous attendent les poèmes de Hossein Sharang, qui rendent un hommage vibrant à la disparue. Puissent ces images et ces mots alimenter votre audace et vous donner accès à des montagnes de courage!

Nous qui n'avons que notre bonne volonté à offrir à des personnes qui, le plus souvent, font face à une issue fatale après une longue histoire de maladie, risquons-nous de perdre toute foi en la validité de notre pratique d'accompagnement si nous nous mettons à scruter de trop près le visage et les armes de ceux qui tuent avec tant d'efficacité? Donner violemment la mort est une pratique qui dure, qui se transmet de génération en génération et dont la suprématie grandissante nous oblige à des constats douloureux.
Dire: «Oui, je suis là», c'est faire un énorme pari. C'est miser sur ce qu'il y a de vivant en moi pour accueillir la vie qui se cherche dans le corps souffrant de l'autre. Il faut avoir une certaine candeur pour croire que ces quelques mots réussiront à changer quelque chose. Pourtant, j'y crois. Dans ma famille, j'ai trouvé dès ma plus tendre enfance des hommes et des femmes désarmés, mais capables de m'apaiser en disant ces mots. Tous ceux et toutes celles qui ont pris soin d'un enfant savent aussi, comme moi, que c'est avec tout son corps bien plus que sa voix qu'il faut apprendre à dire: «Je suis là.» Pour apaiser, tout simplement. Pour en arriver, à même sa propre respiration, à donner de l'espace à l'autre afin qu'il réussisse à s'installer dans son propre rythme. C'est toujours avec cet espoir que j'ai prononcé ces mots auprès de chacune des personnes que j'ai accompagnées pendant la phase terminale de leur cancer. Avec audace, nous y avons cru ensemble. Au chevet des mourants, les hommes et les femmes que je côtoie aujourd'hui sont plus vulnérables que blindés, plus humbles que triomphants même dans l'opulence que permet la technologie. Je sais grâce à eux qu'une authentique qualité de présence à autrui est en train de se transmettre d'une génération à l'autre d'accompagnants, qu'ils soient des proches, des soignants professionnels ou des bénévoles. Attentifs aux périls de l'accompagnement, nous apprenons ensemble à donner la suprématie à ce qui nous fait naître à un présent vivant.

\section{ARRÊTEZ D'EN FAIRE TROP, MADAME, MONSIEUR, ET REPOSEZ-VOUS !}

Ai-je déjà eu le culot d'exiger cela d'une personne que je prétendais accompagner alors que la mort rôdait aux portes? Si je l'ai fait sans même m'en apercevoir, je me promets solennellement aujourd'hui de garder l'œil ouvert pour mettre un frein à mes éventuelles tentatives de sollicitude à la Taliban, ou tout au moins pour faire suivre mes savantes exhortations d'un franc éclat de rire qui confirmera à la personne à qui je m'adresse que ces mots m'ont échappé et que je ne me prends pas trop au sérieux dans 
mon rôle de gardienne de son «bien-faire». Nous en aurons toujours à apprendre sur la meilleure façon de bien faire! Les exigences éthiques de l'accompagnement d'une personne malade ou tout simplement vieillissante ont d'ailleurs inspiré à plusieurs des auteurs que vous lirez dans ce numéro des réflexions qui nous seront précieuses pour nommer les périls de l'accompagnement.

Comment bien faire, malgré le péril du temps qui use. Car elle peut se faire longue la période d'accompagnement! Plus je m'épuise au chevet de l'autre, plus j'aurai besoin qu'il ou elle reste tranquille! Jean-François Malherbe met à contribution les ressources de l'éthique et de la philosophie pour exposer les risques de violence dans la relation d'aide en général et en particulier dans les soins palliatifs. Le temps de l'accompagnement est un temps de crise; souvent même une crise qui n'en finit pas de durer. Or pour qu'une crise puisse ouvrir à un changement, pour qu'elle soit vécue comme un "événement vivifiant plutôt qu'un processus mortifère ", il y a des conditions à respecter, dit Malherbe. Entre autres: «Nul d'entre nous n'aime parler à des murs », nous rappelle-t-il. Du même coup, nous sommes invités à sonder d'un regard interrogateur nos douces pratiques d'accompagnement et les vertueuses exhortations que nous dispensons si généreusement... au péril de devenir, nous-mêmes, un mur qui fait obstacle au cheminement d'autrui et aux efforts qu'il fait pour se remettre au monde, d'une respiration à l'autre.

Quand mes mots s'emploient à faire du bruit autour d'une personne que je prétends accompagner, ne suis-je pas en train de lui demander d'arrêter son propre travail d'être pour s'occuper de mon épuisement, de ma respiration à bout de souffle? Comment croire qu'en se reposant sur commande, ma commande, un être serait mieux préparé à tourner son attention vers le grand souffle, la grande respiration qui déploie en nous, au fil des jours, les puissantes harmonies qui finissent par nous emporter? Car Malherbe ajoute: «Nul d'entre nous n'a le droit d'empêcher que l'autre naisse avant que de mourir. » L'accompagnement en soins palliatifs apparaît dès lors comme un travail d'accouchement mutuel (Laflamme, 1995) pour l'accompagnant et pour l'accompagné-e. L'un comme l'autre, ils sont en travail. Ce qui les attend ne se compare pas, mais ils partagent néanmoins le même étonnement devant la vie qui se donne par des chemins inédits pour constamment nous appeler à naître en elle. Cette personne que j'accompagne, qui sait mieux que moi que l'issue se rapproche, devra-t-elle porter le poids de ma peur de naître en plus de la sienne? Devrat-elle, pour m'épargner, se résoudre à se préparer en cachette à sa propre mort, à ce moment crucial, ce geste ultime qu'il faut à la fois commettre et accueillir en se donnant lentement sa propre posture?

Pour qu'il y ait accouchement mutuel, les mots ne suffiront pas: c'est avec son corps qu'on se met en travail. Et il faudra aussi se préparer aux périls de l'intimité corporelle dont nous parle la sexologue Denise Badeau. Au fil des jours qui passent, accompagnant et accompagné-e en viennent à partager une même intimité. L'accompagnant avait-il vraiment prévu qu'en accédant à l'intimité il lui faudrait recevoir l'autre «dans son propre territoire intime sans se sentir envahi, perturbé, bousculé ou contaminé»? Badeau nous prévient: lors de cette mise à l'épreuve, «on peut se découvrir bien différent de ce qu'on croyait, moins compétent, moins généreux, surtout moins détaché». Il n'est pas toujours facile de garder la bonne distance quand on se retrouve ainsi, par la force des choses, «sensoriellement présent» à ce que vit l'autre dans son corps, constamment dans l'urgence de «le regarder, le sentir, être attentif aux sons qui émanent de sa vie», trop souvent avec la crainte de ne pas être à la hauteur au moment où son corps malade ou vieillissant va flancher et où il aura le plus besoin de nous. Mais adopter la posture héroïque de l'accompagnant qui se sacrifie pour l'autre, ce sera tôt ou tard se retrouver aigri et celui ou celle qui nous avait laissé pénétrer dans son intimité se sentira trahi-e. La présence amie qui rend la vie plus légère au quotidien se doublera d'une ombre: celle d'un créancier de plus à satisfaire de façon à ne jamais perdre le certificat de bonne conduite qui garantit la poursuite de la relation.

Pour faire suite à la description des périls du toucher que nous propose Denise Badeau à partir de sa longue pratique dans les milieux d'intervention, vous voudrez lire le texte qu'a inspiré à Martyne-Isabel Forest le récent film d'Almodovar, Parle avec elle. Sous prétexte de donner voix à Alicia, l'un des personnages du film, Forest nous donne à voir ce que c'est que de se vivre et de se dire comme la personne qui a besoin d'être accompagnée. Son regard se fait tour à tour candide et noir pour nous faire partager de l'intérieur ce que le grand écran ne montrait pas. Un rude parcours qui révèle que l'accompagnement est à la fois souhaité lorsqu'il donne accès à une présence qui comble des attentes, et rejeté lorsqu'il impose une présence dont on ne peut se déprendre. Ce qui nous est ainsi révélé en gros plans, c'est une vulnérabilité qui à la fois nous ouvre à la vie et freine nos élans vers elle.

Dans la force de l'âge, nous nous entraînons sans relâche à baliser et à réclamer nos champs d'autonomie. Cela fait de nous des personnes énergiques, dont on dit avec respect qu'elles savent ce qu'elles veulent. Lorsque nous serons handicapés ou vieillissants, nous serons classés dans la catégorie précisément nommée: «en perte d'autonomie». Cela nous transformera-t-il en victimes face à ce que voudront pour nous des intervenants plus autonomes que nous? De retour à la philosophie avec Alberto Bondolfi, nous explorons les périls qu'il y a à conjuguer le verbe «être autonome» avec l'horizon d'impuissance qui caractérise le fait de devoir «être accompagné» dans la maladie et dans le trépas. Le constat est troublant: "La solitude de la maladie, et surtout celle du trépas, ne peut être surmontée par aucune bienfaisance, même la plus empathique.» Bondolfi présente dans ce contexte deux interprétations de notre devoir de solidarité envers autrui: l'une qui souligne ce que nous avons en commun avec l'accompagné-e, l'autre qui montre le décalage qui est présent dans toute relation d'aide à cause de ce qu'il y a de différent entre les personnes qui accompagnent et celles qui sont accompagnées. Pour en arriver à « une bonne conjugaison avec le principe d'autonomie», Bondolfi nous invite à miser sur une solidarité avec autrui «qui souligne la différence plutôt que la destinée commune». Selon lui, c'est sur cette voie que la réflexion bioéthique en arrivera à découvrir un équilibre plus harmonieux.

Les débats contemporains sur «la façon la plus adéquate d'organiser les phases finales de la vie humaine, les propositions de législation autour des pratiques euthanasiques ou bien liées à l'aide au suicide» s'articulent selon Bondolfi autour de deux principes, celui d'autonomie et celui de bienfaisance. Avec le texte d'Éric Volant, nous poursuivons la réflexion sur de telles questions qui mettent la solidarité en débat. Volant nous invite à donner à la personne soignée une position de partenaire dans la gérance des soins: "Liés à la personne mourante en fraternité solidaire, les soignants, les proches et les bénévoles forment, en principe, un partenariat 


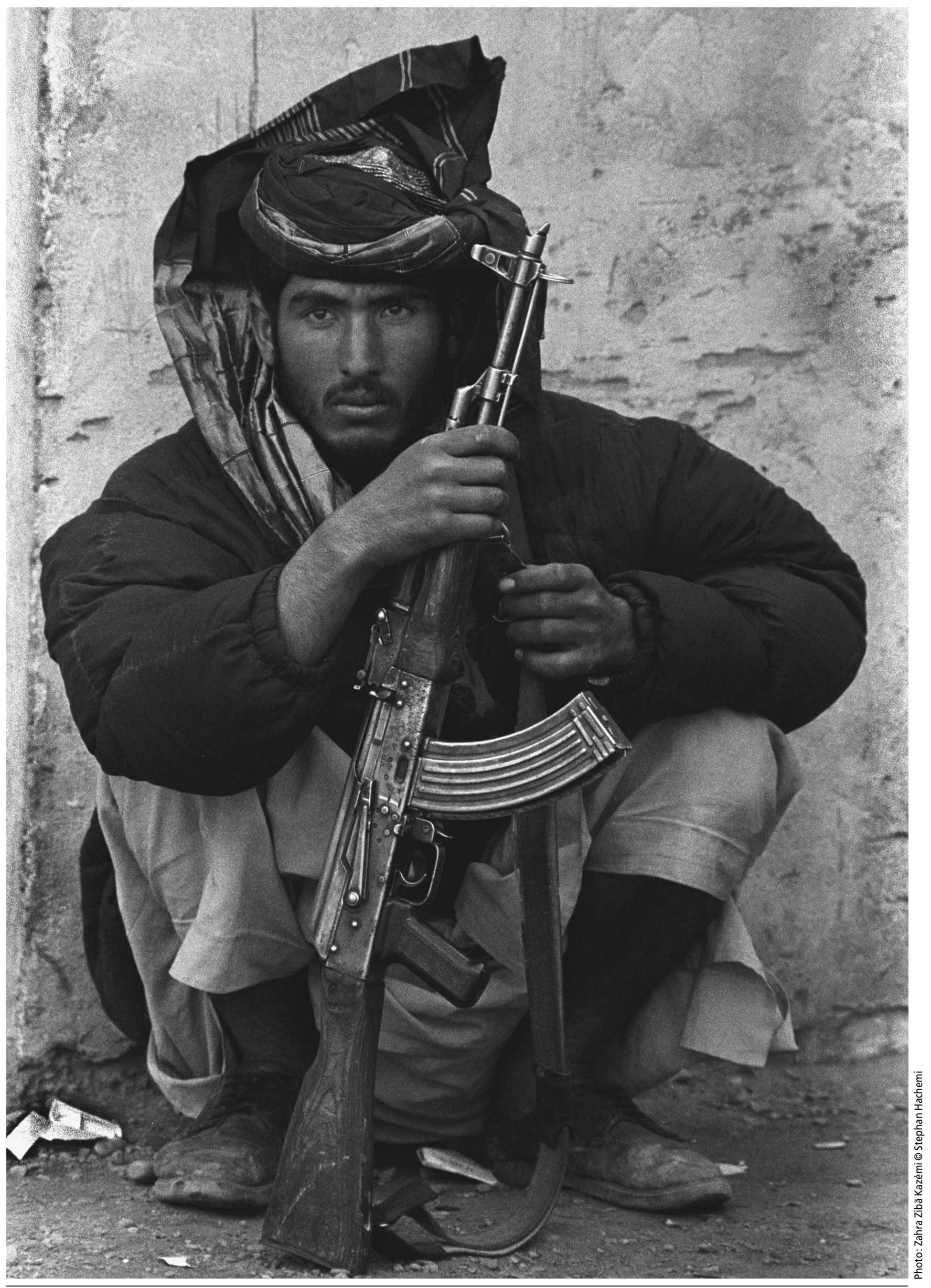


et, par conséquent, ils sont en mesure de se créer des lieux de réflexion en équipe sur le sens de l'acte de mourir et ses frontières». Il plaide pour que soient accomplis dans la bonté et la générosité les gestes qui permettront de respecter les volontés ultimes d'une personne mourante lorsqu'elle considère avoir atteint «le seuil». Il y aura un péril de la solidarité dans la construction du sens car, ajoute-t-il: «Face aux problèmes d'ordre éthique, situés au seuil des limites du sens, les partenaires impliqués ont tendance à durcir leurs positions au nom de la loi ou de la religion. »Volant conclut d'ailleurs en rappelant "l'urgence d'avis d'ordre légal pour encadrer et soutenir les pratiques médicales au chevet des mourants».

\section{VIVRE LE TIRAILLEMENT ENTRE ENGAGEMENT ET RÉSISTANCE}

Plus que devant les tribunaux, c'est encore dans les familles que se vivent chez nous les miracles et les périls de l'accompagnement. À partir d'une recherche qu'elle avait effectuée auprès d'enfants adultes qui prenaient soin d'un parent âgé et malade, Jacynthe Pépin (1996) avait déjà réussi à décrire l'expérience de l'aidant naturel comme un «mouvement entre l'engagement et la résistance». Bref, choisir de le faire, tout en sachant qu'on est en quelque sorte désigné ou choisi pour le faire. C'est cette dynamique paradoxale qui alimente le lien entre le parent malade ou vieillissant et l'enfant adulte qui accepte le rôle d'aidant naturel. En analysant les témoignages recueillis auprès des personnes rencontrées dans le cadre de sa recherche, Pépin avait dégagé trois éléments pour expliquer l'émergence d'un tel tiraillement: l'impression d'être le seul ou la seule à se préoccuper du parent dans un contexte où il y a peu d'alternatives satisfaisantes de soin, l'impression que personne (incluant les professionnels de la santé) ne sait comment soulager la souffrance du parent dans un contexte où les soins professionnels mettent l'accent sur les aspects physiques de la maladie, et enfin l'impression de ne pas avoir de liberté ni de vie à soi, dans un contexte où le rôle d'aidant naturel est peu valorisé. Les trois éléments de contexte qui nuancent chacune des affirmations ont peu changé depuis. Encore aujourd'hui, l'enfant adulte qui choisit de dire «Oui, je suis là » à son parent âgé ou qui a besoin de soins se retrouve d'office choisi (par qui? par le système?) pour répondre non plus seulement «à » son parent mais pour répondre «de» tout ce qui va se passer dans la famille et à l'extérieur de la famille au cours de l'évolution de la dyade qu'il forme maintenant avec son parent malade ou vieillissant. Les avocates Dominique Demers et Lucie Legault ont rassemblé pour nous des données qui permettent de préciser dans quelle situation se trouvent présentement au Canada les trois millions de personnes qui jouent un rôle de proches aidants auprès d'un parent ou d'un ami. On constate que les aidants naturels sont encore en équilibre précaire entre une aide publique qui se donne au comptegouttes et une présence toujours lourde à assumer mais qui ne se refuse pas.

Ce sont d'autres «tensions contradictoires» que dévoile la recherche menée récemment par Suzanne Mongeau, Manon Champagne et Pauline Carignan auprès de bénévoles qui accompagnent des familles qui prennent soin d'un enfant atteint d'une maladie à issue fatale. L'expérience des bénévoles qui participent à ce programme de répit à domicile dévoile ici encore une dynamique paradoxale. Il s'agit de jeunes enfants et les échéances sont terribles. Comment espérer abolir les tensions? On cherchera plutôt à les assumer, peut-être même à se jouer d'elles... d'autant plus que les enfants, nos partenaires de jeu, nous gardent dans l'intensité du présent.

Laccompagnement qui se vit auprès d'une famille peut aussi prendre la forme d'un parcours qui nécessite plusieurs années avant que tous les acteurs de la tragédie puissent faire une entrée en scène. L'expérience que vivent les intervenants du Centre maternel et infantile sur le sida de l'Hôpital Sainte-Justine les amène à reconnaître le rôle stratégique du temps. Guylaine Morin et Johanne Samson nous permettent d'entrevoir ce qui se passe quand le VIH/sida frappe dès les premiers instants d'une jeune vie et s'installe à demeure. À mesure que l'enfant grandit, les défis de l'accompagnement se concrétisent et la famille se retrouve à la fois au centre du problème et de sa résolution. Les chercheures ont pu constater comment le tiraillement des acteurs se traduit douloureusement dans la difficulté de prendre la parole pour dire le diagnostic. Comment accompagner une parole qui sait qu'elle va blesser, comment l'aider à découvrir qu'elle saura aussi dire la tendresse et le courage d'aider la vie à suivre son cours?

Quand la famille n'est pas là pour fournir des aidants naturels, à qui faut-il s'adresser pour prendre le relais? Au Québec, ce sont «majoritairement des femmes du grand âge» qui ont besoin de ce relais: elles habitent présentement dans nos résidences pour personnes âgées. Michèle Charpentier a interrogé dans le cadre de sa recherche des intervenants du réseau privé et du réseau public qui accompagnent ces personnes souvent fragilisées; il s'agissait de propriétaires de résidences privées, de gestionnaires publics des services de soutien à domicile et d'hébergement ainsi que de travailleurs sociaux impliqués dans l'orientation et la relocalisation des personnes âgées. Les données qu'elle a obtenues à la suite de l'envoi d'un questionnaire par la poste et les commentaires qu'elle a recueillis lors de groupes de discussion débouchent sur une analyse qui s'intéresse à la fois «aux enjeux sociaux, éthiques et politiques» associés à ce milieu d'intervention. Les périls nommés dans l'article sont à maints égards désespérants. Puisqu'il s'agit d'un contexte marchand, retenons les périls liés à la liberté contractuelle: «En ce qui a trait au principe du choix libre et éclairé de sa résidence, de la liberté contractuelle, les intervenants rencontrés ont exprimé d'importantes réserves. Les témoignages relatant les circonstances entourant la sélection de leur futur milieu de vie sont éloquents. Selon plusieurs interlocuteurs, ce sont les enfants, surtout les filles, qui choisissent la résidence. Il arrive que la personne âgée n'ait même pas visité les lieux avant d'emménager.» L'expression «en perte d'autonomie» prend ici tout son sens et fait pâlir les consolations que nous avions cru trouver dans la philosophie!

Démunis face aux répercussions tragiques du tiraillement que nous vivons entre engagement et résistance, saurons-nous prendre le risque de l'entraide pour combler les défaillances de la liberté contractuelle? C'est un éloquent plaidoyer en faveur de l'entraide que nous livre Flore FournelleLe Buis en retraçant le parcours qui l'a amenée à créer en Suisse le groupe Virage, à l'intention des personnes atteintes de cancer et de leurs proches. Ce groupe permet une forme d'accompagnement mutuel depuis plus de 20 ans déjà en mettant à contribution des accompagnants qui ont eux-mêmes fait l'expérience de la maladie et qui connaissent donc le parcours dans lequel s'engagent les accompagnés-es. L'expérience de ces soignés devenus accompagnants leur a permis de nommer clairement les besoins qui n'avaient pas trouvé leur réponse: «transport, stationnement, horaires, stress, sentiment d'isolement et souvent de solitude, etc.». Des besoins bien ordinaires, qui appellent une réponse 
concrète plutôt que des discours. Des besoins bien ordinaires sous lesquels se cache une expérience dramatique. Certains voudront aussi compter sur un ressourcement spirituel pour faire face à ce drame. Les outils bien concrets que nous propose la pasteure Cosette Odier pourront surprendre car on se complaît souvent dans un certain flou lorsqu'il faut parler d'accompagnement spirituel. Cosette Odier se pose des questions précises dans l'espoir de mieux comprendre le vécu de l'accompagné-e et elle nous prévient que le croyant «fait vivre à l'accompagnant bien des paradoxes».

Choisir de s'engager dans l'accompagnement tout en résistant à cet engagement pour lequel on est choisi malgré soi, c'est ainsi que nous est d'abord apparu le paradoxe vécu par l'enfant adulte qui accompagne son parent vieillissant. Dans la grande famille humaine, nous n'avons pas choisi notre place mais nous la re-choisissons à chaque matin; peut-être en viendrons-nous à découvrir que c'est un privilège de vivre un tel paradoxe.

\section{DIRE: «OUI, JE SUIS LÀ», AU-DELÀ DES GRIEFS ET AVANTAGES}

Nos auteurs ont aussi puisé aux ressources que nous offrent la littérature, le cinéma et la ritualité contemporaine pour alimenter leur réflexion sur l'accompagnement. Maurice Boutin nous parle du récit publié par Maurice Blanchot en 1953 sous le titre Celui qui ne m'accompagnait pas. La négation dans le titre de l'ouvrage est provocante. Elle réussit encore aujourd'hui à «déranger cette rumeur autour de nous», une rumeur au sujet de ce que Boutin appelle le mythe de l'accompagnement «alimenté par la mystique du compagnon». Voudrons-nous ranger ce mythe et cette mystique au rang des périls? Boutin considère pour sa part que cette provocation permet de lever une équivoque: «celle qui se contente de faire de l'accompagnement une activité et un comportement parmi d'autres possibles, un sujet dont on disserte comme on le ferait pour n'importe quoi, ou encore un symptôme par exemple de l'amour, ou simplement (?) de l'amitié, de la solidarité entre mortels». Dans le vocabulaire du choisir, l'exigence d'accompagnement devenait un paradoxe inconfortable. Boutin remplace le tiraillement entre choisir et être choisi par trois mots séduisants: attirer sans obliger. Il écrit: «Plus qu'aucune morale, l'exigence d'accompagnement attire tout être humain, en même temps qu'elle n'oblige en rien et ne fait grief ni avantage de rien ». Comment faire grief, tirer profit, dresser un bilan de l'accompagnement lorsque s'impose à nous ce que Blanchot appelle «la démesure du mouvement de mourir» ? Boutin ajoute à notre plaisir en nous proposant aussi deux extraits du livre de Blanchot intitulé L'amitié. Lire ces quelques lignes, c'est se laisser séduire!

Nathalie Parent a choisi de nous présenter dans son article deux romans de Marguerite Yourcenar qui font contraste et qui mettent en scène «une fin accompagnée et l'autre non ». Dans les deux romans, le personnage principal est un peintre. Cornélius Berg, dont l'histoire se déroule en Occident au XVII ${ }^{\mathrm{e}}$ siècle, fait route seul; Wang-Fô, qui vit en Chine pendant l'empire des Han, est accompagné par un élève qui en vient à le devancer dans la mort. C'est avec fascination que l'on assiste à l'émergence des images, développées en positif et en négatif, à travers lesquelles se dévoilent d'autres formes de cette mystique du compagnon aussi évoquée par Maurice Boutin.

La romancière Denise Blais nous revient avec un autre de ses très beaux textes. On y parle d'une double perte et du même constat d'un accompagnement impossible: aujourd'hui, il y a eu un suicide chez les voisins, sans commentaires; il y a trente ans déjà, il y avait eu un accident chez nous, sans commentaires. Le texte jette une lumière crue sur des gens qui «font semblant». Ce n'est pas pour leur en faire grief; c'est pour libérer le «hurlement muet» qui monte dans la gorge et pour se battre contre «le silence de l'enfance plus fort que tout!»

Armelle Chitrit alterne entre la prose et la poésie pour mieux dire les mains. Sous sa plume, ce symbole si lourdement investi reprend une légèreté inédite. La main qui accompagne ouvre «à la possibilité de recevoir une parole (parlante) comme un temps s'ajoutant au temps », écrit-elle. En disant: «Oui, je suis là », gardons l'espoir d'habiter encore un peu ce temps, qui ne peut surgir que de nous pour exister entre nous.

Des belles lettres aux belles images, suivons Joseph J. Lévy qui retrace à partir de l'histoire du cinéma «quelques jalons de la problématique des soins et de l'accompagnement». Les étapes parcourues depuis 1916 révèlent à la fois des stéréotypes et des avancées audacieuses. En fin de parcours, nous retrouvons à nouveau le film d'Almodovar Parle avec elle, mis à contribution pour illustrer les enjeux de l'accompagnement en milieu hospitalier.

En nous laissant interpeller par le biais d'une nouvelle forme de ritualité, nous aurons l'occasion d'aborder, quoique trop brièvement, l'accompagnement dans le deuil. François Gauthier nous fait partager l'expérience qu'il a vécue en 2003 au Nevada, lors du Festival Burning Man. Les noms des lieux de célébration que nous découvrons grâce à lui évoquent des réalités qui nous sont familières (le temple de l'Honneur, le temple des Pleurs, le temple de la Joie), mais il en est tout autrement des conduites rituelles qu'il décrit et qui vont « du festif le plus sauvage au recueillement le plus profond». Si l'univers qui se déploie pendant les sept jours du festival naît d'un besoin d'être ensemble pour dire sa peine, de créer des lieux collectifs pour accueillir le deuil, la démarche est polarisée dès le départ par une décision qui fait consensus: mettre le feu, laisser brûler ce qui se trouve là. Nul besoin alors de se livrer à un calcul pointilleux des griefs et des avantages. Dans la frénésie, les comptes sont réglés. Est-ce encore la mystique du compagnon qui s'exprime sous une autre forme lorsqu'on brûle l'effigie humaine qui donne son nom à ce festival?

En fin de parcours, nous nous découvrons à la fois compagnons de misère et âme solitaire. Plus souvent essoufflés que ravis, nous n'avons pas le temps qu'il faudrait pour faire grief ou avantage de quoi que ce soit. Au péril de l'accompagnement, nous sommes livrés à ce qui nous dépasse sans cesse, même et encore plus quand il nous rejoint au plus près.

\section{Bibliographie}

LAFLAMME, Diane (1995). «Le corps humain en travail: travail d'enfantement de soi et d'accouchement mutuel dans la relation de soins et l'accompagnement des mourants» dans Revue de l'Association pour la recherche qualitative, Vol. 12, hiver 1995, p. 27-38; www.recherche-qualitative.qc.ca.

PÉPIN, Jacynthe (1996). «L'ethnographie interprétative centrée sur une expérience, celle du soin (caring) auprès d'un parent âgé» dans Recherches qualitatives, Vol. 15, p. 73-87; www.recherchequalitative.qc.ca. 